\title{
GEOMETRIC REALIZATIONS OF REPRESENTATIONS OF FINITE LENGTH II
}

\author{
Charles H. Conley
}

Let $G=H \times{ }_{s} \mathbb{R}^{n}$ be a semidirect product Lie group, let $\mathcal{O}$ be a locally closed orbit of $H$ in the dual of $\mathbb{R}^{n}$, and let $S$ be the subgroup of $H$ stabilizing some point of $\mathcal{O}$. Suppose that $\mathcal{U}$ is a representation of length $n+1$ of $G$, such that every irreducible representation in the composition series of $\mathcal{U}$ is associated to the orbit $\mathcal{O}$ and a finite dimensional representation of $S$ by the Mackey machine. We prove that if $H$ is a real linear algebraic group, $S$ is an algebraic subgroup of $H$, and all finite dimensional representations of $S$ are rational, then $\mathcal{U}$ may be realized as a subquotient of the canonical representation of $G$ in the space of functions on the $n^{\text {th }}$-order infinitesimal neighborhood of $\mathcal{O}$ in its ambient vector space, taking values in some finite dimensional representation of $H$.

\section{Introduction.}

In this paper we complete the program of finding geometric realizations of indecomposable representations of real semidirect product Lie groups, begun in [3], [4], and [5]. Fix a semidirect product Lie group $G=H \times{ }_{s} A$ formed by a real Lie group $H$ acting linearly on a real vector group $A$, and assume that the orbits of the dual action of $H$ on $A^{*}$ are locally closed. Given such an orbit $\mathcal{O}$, let $p_{0}$ be a point of $\mathcal{O}$ and let $S$ be the (closed) subgroup of $H$ stabilizing $p_{0}$. Given any representation $\sigma$ of $S$ in a finite dimensional complex vector space $V$, let $\operatorname{Ind}_{S}^{H} \sigma$ be the representation of $H$ induced by $\sigma$ in the space $C_{c}^{\infty}\left(\mathcal{O}: H \times{ }_{S} V\right)$ of smooth compactly supported sections of the $H$ vector bundle over $\mathcal{O}$ associated to $V$. For any element $p$ of $A^{*}$, let $e^{i p}$ denote the one dimensional representation of $A$ with character $a \mapsto \exp i\langle a, p\rangle$, and note that $\sigma \otimes e^{i p_{0}}$ is a representation of the inhomogeneous stabilizer $S \times{ }_{s} A$ in the space $V$, because $S$ stabilizes $p_{0}$. We will study representations of finite length of $G$ having a topologically split composition series of representations of the form $\operatorname{Ind}_{S A}^{G}\left(\sigma \otimes e^{i p_{0}}\right)$, and we will always view this representation as acting in the space $C_{c}^{\infty}\left(\mathcal{O}: H \times{ }_{S} V\right)$, where the action of $H$ is the induced one and the action of $a \in A$ is multiplication by the restriction to $\mathcal{O}$ of the character function $\xi_{a}: A^{*} \rightarrow \mathbb{C}$ defined by $\xi_{a}(p)=\exp i\langle a, p\rangle$. 
Guichardet has proven that there are no indecomposable representations of $G$ of this type having in their composition series representations associated to more than one orbit [6], and so we may restrict our attention to the category $\operatorname{Ext}_{G} \mathcal{O}$ of smooth topologically split representations of $G$ whose composition series elements are all induced from the stabilizer of a fixed orbit $\mathcal{O}$. This category is precisely defined in Section 1.

Rideau was the first to discover that not all representations in $\operatorname{Ext}_{G} \mathcal{O}$ are induced, because the elements of $H$ may act by differential operators above their action on the orbit $\mathcal{O}$, instead of by bundle maps above their action on $\mathcal{O}$ as for induced representations [8]. Thus there arose the problem of describing these representations in some general way, analogous to the description of the irreducible representations given by the Mackey machine, to which this paper gives a solution: assuming that $H$ is a real linear algebraic group, $S$ is an algebraic subgroup of $H$, and all finite dimensional representations of $S$ are rational, we prove that any representation in $\operatorname{Ext}_{G} \mathcal{O}$ may be realized as a subquotient of the canonical representation of $G$ in the space of smooth compactly supported functions on an infinitesimal neighborhood of $\mathcal{O}$ in the ambient space $A^{*}$, taking values in a finite dimensional representation of $H$. As far as we know, the first example of such a realization was given by Cassinelli, Truini, and Varadarajan in [2].

We remark that in [7] Guichardet gives a complete solution to the general problem posed by Rideau, in the case that the tangent bundle $T \mathcal{O}$ of the orbit admits an $H$-covariant complement in the untwisted bundle $\mathcal{O} \times A^{*}$ : here all representations in $\operatorname{Ext}_{G} \mathcal{O}$ are induced. In the case of the Poincare group, only the light cones fail to satisfy this criterion; it was these orbits which Rideau originally studied.

Our results are organized as follows. In Section 1 we recall the definitions of $\operatorname{Ext}_{G} \mathcal{O}$ and functions on infinitesimal neighborhoods from [5], state our main result, show that it follows from Section 2 and our earlier papers, and treat the example of the Poincare group. In Section 2 we recall from [5] the definition of representations of the infinitesimal neighborhood of $S$ in $H$, or "representations of $S^{\infty}$," and in the case that $S \subset H$ are algebraic matrix groups we prove that any representation of $S^{\infty}$ may be realized as a subquotient of the restriction of a representation of $H$.

We would like to thank V.S. Varadarajan for leading us to this problem, David Witte and Bruce Crauder for helpful discussions, and Oklahoma State University for their support during the course of the work.

\section{Definitions, the Main Result, and an Example.}


Throughout this paper fix $G=H \times{ }_{s} A$ and $\mathcal{O}, p_{0}$, and $S$ as in the introduction. In this section we recall some definitions from [5], and state our main result. We will use Schwartz' notations $\mathcal{D}$ and $\mathcal{E}$ in place of $C_{c}^{\infty}$ and $C^{\infty}$, and if $B$ is a vector bundle over a space $\mathcal{O}$ then $\mathcal{D}(B)$ and $\mathcal{E}(B)$ will always denote the sections of $B$, equipped with their usual topologies of uniform convergence of all derivatives on all compact sets. We will write $\mathcal{D} B$ instead of $\mathcal{D}(B)$ whenever it simplifies the notation, and similarly for $\mathcal{E}$. We will write $B(p)$ or $B_{p}$ for the fiber of $B$ at $p \in \mathcal{O}$. All representations and spaces of functions are complex unless otherwise noted.

Definition. Let $\operatorname{Ext}_{G} \mathcal{O}$ be the category of smooth representations of $G$ in topological vector spaces $V$ that admit a $G$-invariant flag

$$
V=V_{0} \supset \cdots \supset V_{n} \supset V_{n+1}=0
$$

with the following properties. First, for each $i$ there is a closed subspace of $V_{i}$ complementary to $V_{i+1}$. Second, each subquotient $V_{i} / V_{i+1}$ is topologically equivalent to the representation $\operatorname{Ind}_{S A}^{G}\left(\sigma \otimes e^{i p_{0}}\right)$ for some finite dimensional complex representation $\sigma$ of $S$. Morphisms are continuous linear intertwining maps.

Next we define the space $\mathcal{D O} \mathcal{O}^{(n)}$ of functions on the $n^{\text {th }}$ order infinitesimal neighborhood $\mathcal{O}^{(n)}$ of $\mathcal{O}$ in its ambient space $A^{*}$, although in fact we will not define $\mathcal{O}^{(n)}$ itself, but only think of it heuristically as a space. We will see that $\mathcal{D O} \mathcal{O}^{(n)}$ carries a canonical representation $\mathcal{V}^{(n)}$ of $G$ that is an element of $\operatorname{Ext}_{G} \mathcal{O}$. All of the following definitions and statements are discussed in more detail in [5].

Suppose that $N$ is a regularly imbedded submanifold of an arbitrary smooth manifold $M$. Let $J^{n} M$ be the $n^{\text {th }}$ jet bundle of $M$, the fibers of which are algebras, and let $j_{n}: \mathcal{D} M \rightarrow \mathcal{D} J^{n} M$ be the $n$-jet map, which is an injective algebra homomorphism. Note that the sections $\mathcal{D}\left(\left.J^{n} M\right|_{N}\right)$ of the restriction of the bundle $J^{n} M$ to $N$ also form an algebra.

Definition. The space $\mathcal{D} N^{(n)}$ of smooth compactly supported functions on the $n^{t h}$-order infinitesimal neighborhood $N^{(n)}$ of $N$ in its ambient space $M$ is the subspace of $\mathcal{D}\left(\left.J^{n} M\right|_{N}\right)$ consisting of sections $F$ such that for each $p \in N$, there is an open neighborhood $V$ of $p$ in $M$ (not $N$ ) and a smooth function $f_{V}$ on $V$, such that for all $q \in V \cap N$ we have $j_{n} f_{V}(q)=F(q)$.

So locally, functions on $N^{(n)}$ are restrictions to $N$ of $n$-jets of functions on $M$. The space $\mathcal{D} N^{(n)}$ is a closed subalgebra of $\mathcal{D}\left(\left.J^{n} M\right|_{N}\right)$, and so it is an inductive limit of Frechet spaces. We remark that it admits partitions of unity. For $k \leq n$, let $\pi_{k n}: J^{n} M \rightarrow J^{k} M$ be the restriction map, which is an algebra homomorphism on the fibers. Then $\pi_{k n}\left(\mathcal{D} N^{(n)}\right) \subset \mathcal{D} N^{(k)}$, and we 
write $I_{k n}$ for the kernel of $\pi_{k n}: \mathcal{D} N^{(n)} \rightarrow \mathcal{D} N^{(k)}$. We have the flag of ideals

$$
\mathcal{D} N^{(n)} \supset I_{0, n} \supset I_{1, n} \supset \cdots \supset I_{n-1, n},
$$

and it turns out that $I_{k n}$ admits a (non-canonical) closed complementary subspace in $I_{k-1, n}$. Furthermore, the quotient $I_{k-1, n} / I_{k, n}$ is canonically isomorphic to the subspace $I_{k-1, k}$ of $\mathcal{D} N^{(k)}$, which is itself naturally a $\mathcal{D} N$ module that is canonically isomorphic to the space of sections $\mathcal{D}\left(\mathcal{S}^{n} T^{\perp} N\right)$ of the $n^{\text {th }}$ symmetric power $\mathcal{S}^{n}$ of the conormal bundle $T^{\perp} N \subset \Omega^{1} M$ of $N \subset M$. Therefore $\mathcal{D} N^{(n)}$ is linearly homeomorphic to $\oplus_{0}^{n} \mathcal{D}\left(\mathcal{S}^{i} T^{\perp} N\right)$.

Since $\mathcal{O}$ is a locally closed orbit of the Lie group $H$ in $A^{*}$, it is regularly imbedded, and so we may consider the case that $N=\mathcal{O}$ and $M=A^{*}$. Here the $H$-action on $A^{*}$ defines an $H$-bundle structure on $J^{n} A^{*}$, such that $j_{n}$ intertwines the representations of $H$ on $\mathcal{D} A^{*}$ and $\mathcal{D} J^{n} A^{*}$. Since $\mathcal{O}$ is $H$-invariant, there is a smooth representation of $H$ on $\mathcal{D}\left(\left.J^{n} A^{*}\right|_{\mathcal{O}}\right)$, and one finds that $\mathcal{D} \mathcal{O}^{(n)}$ is an invariant subspace. We define $\mathcal{V}^{(n)}$ to be the resulting representation on $\mathcal{D O} \mathcal{O}^{(n)}$, and we extend $\mathcal{V}^{(n)}$ to a representation of $G$ by letting $\mathcal{V}_{a}^{(n)}$ be multiplication by $\left.\left(j_{n} \xi_{a}\right)\right|_{\mathcal{O}}$ for all $a \in A$, where $\xi_{a}$ is the character function from the introduction. We remark that the representation of $H$ on $\mathcal{D}\left(\left.J^{n} A^{*}\right|_{\mathcal{O}}\right)$ can also be extended to $G$ in this way, and the resulting representation is induced and contains $\mathcal{V}^{(n)}$ as a subrepresentation. However, $\mathcal{V}^{(n)}$ itself is not induced because $\mathcal{D} \mathcal{O}^{(n)}$ is not the space of sections of any subbundle of $\mathcal{D}\left(\left.J^{n} A^{*}\right|_{\mathcal{O}}\right)$.

In this setting the above maps $\pi_{k n}$ are $G$-maps, and so the ideals $I_{k n}$ of $\mathcal{D O} \mathcal{O}^{(n)}$ are $G$-invariant. Thus $\mathcal{V}^{(n)}$ admits the $G$-flag

$$
\mathcal{D O} \mathcal{O}^{(n)} \supset I_{0, n} \supset \cdots \supset I_{n-1, n} .
$$

Furthermore, the conormal bundle $T^{\perp} \mathcal{O}$ is an $H$-bundle and the isomorphism from $I_{k-1, n} / I_{k, n}$ to $\mathcal{D}\left(\mathcal{S}^{k} T^{\perp} \mathcal{O}\right)$ is a $G$-map, and so we have the following proposition.

Proposition 1.1. The representation $\mathcal{V}^{(n)}$ of $G$ on $\mathcal{D O}^{(n)}$ is an object of $\operatorname{Ext}_{G} \mathcal{O}$, whose composition series is isomorphic to $\mathcal{D}\left(\mathcal{S}^{k} T^{\perp} \mathcal{O}\right), 0 \leq k \leq n$.

If $\pi$ is a finite dimensional representation of $H$ on a space $V$, we may extend it to a representation of $G$ that is trivial on $A$. Then $\operatorname{Ext}_{G} \mathcal{O}$ is closed under tensoring with $\pi$, and so it contains the representation $\mathcal{V}^{(n)} \otimes \pi$ on the space $\mathcal{D} \mathcal{O}^{(n)} \otimes V$ of $V$-valued functions on $\mathcal{O}^{(n)}$. Our main result is as follows.

Theorem 1.2. Suppose that $H$ may given the structure of a real algebraic matrix group, such that $S$ is an algebraic subgroup whose finite dimensional 
representations are all rational. Then any representation of length $n+1$ in the category $\operatorname{Ext}_{G} \mathcal{O}$ is a subquotient of $\mathcal{V}^{(n)} \otimes \pi$, for some finite dimensional representation $\pi$ of $H$.

Proof. We will show that the theorem follows from the results of [3], [4], and [5], along with Theorem 2.1 of this paper. We use the notation of [5], which contains a summary of [3] and [4].

In [3] we defined a subcategory $\operatorname{Ext}_{G}^{C} \mathcal{O}$ of $\operatorname{Ext}_{G} \mathcal{O}$ and proved that inclusion is an equivalence of categories, and so it is enough to prove the theorem for any representation in $\operatorname{Ext}_{G}^{C} \mathcal{O}$. In [4] we defined an equivalence of categories $\mathcal{F}$ from $\operatorname{Ext}_{G}^{C} \mathcal{O}$ to a certain category $\mathcal{C}$ of finite dimensional representations of the inhomogeneous stabilizer $S^{\prime}=S \times{ }_{s} A$, where the objects of $\mathcal{C}$ are given with some additional structure which makes them what we call representations of the infinitesimal neighborhood of $S^{\prime}$ in $G$, or representations of $S^{\prime \infty}$. These are defined in Section 2 of this paper. Any representation of $S^{\prime \infty}$ may be restricted to a representation of the infinitesimal neighborhood of $S$ in $H$, or a representation of $S^{\infty}$, and representations of $H$ may also be restricted to $S^{\infty}$. The category of representations of $S^{\infty}$ admits subquotients, and the main result of [5] is that if $\mathcal{U}$ is a representation of length $n+1$ in $\operatorname{Ext}_{G}^{C} \mathcal{O}$, and the restriction to $S^{\infty}$ of the representation $\mathcal{F U}$ of $S^{\prime \infty}$ is a subquotient of the restriction to $S^{\infty}$ of some representation $\pi$ of $H$, then $\mathcal{U}$ is a subquotient of $\mathcal{V}^{(n)} \otimes \pi$.

Now any representation of $S^{\infty}$ may be restricted to a representation of $S$, and in Theorem 2.1 we will prove that if $\sigma$ is a representation of $S^{\infty}$ whose restriction to $S$ is rational, then $\sigma$ is the subquotient of the restriction of some representation of $H$ to $S^{\infty}$. This will prove Theorem 1.2, and also the following stronger variation of it.

Corollary 1.3. Suppose that $S \subset H$ are algebraic matrix groups, but that not all finite dimensional representations of $S$ are necessarily rational. Retaining the notation of Theorem 1.2 and its proof, let $\mathcal{U}$ be a representation of length $n+1$ in $\operatorname{Ext}_{G}^{C} \mathcal{O}$ such that the restriction to $S$ of the representation $\mathcal{F U}$ of $S^{\prime \infty}$ is rational. Then $\mathcal{U}$ is a subquotient of $\mathcal{V}^{(n)} \otimes \pi$ for some representation $\pi$ of $H$.

Example. In his original study of $\operatorname{Ext}_{G} \mathcal{O}$, Rideau considered the case that $G$ is the Poincare group $\operatorname{SL}(2, \mathbb{C}) \times{ }_{s} \mathbb{R}^{1,3}$. Here the stabilizer of each orbit is an algebraic subgroup of $\mathrm{SL}(2, \mathbb{C})$, isomorphic to either $\mathrm{SU}(2), \mathrm{SL}(2, \mathbb{R})$, the entire group $\mathrm{SL}(2, \mathbb{C})$, or the solvable group $\mathrm{S}^{1} \times_{s} \mathbb{C}$. It is well-known that the finite dimensional representations of the first three of these groups are all rational, and it is shown in [1] that this is also true of $S^{1} \times{ }_{s} \mathbb{C}$. Therefore if $\mathcal{O}$ is any orbit of the Poincare group $G$, every representation in $\operatorname{Ext}_{G} \mathcal{O}$ is isomorphic to a subquotient of $\mathcal{V}^{(n)} \otimes \pi$ for some $n$ and $\pi$ as above. 


\section{Representations of Infinitesimal Neighborhoods of Subgroups.}

In this section we recall from [5] the definition of representations of $S^{\infty}$, and prove Theorem 2.1, the result used in the proof of Theorem 1.2. Henceforth we will use gothic letters for complexified Lie algebras of real Lie groups, and we will write $\mathfrak{U}(\mathfrak{k})$ and $\mathfrak{U}_{r}(\mathfrak{k})$ for the universal enveloping algebra of a Lie algebra $\mathfrak{k}$ and its standard filtration, respectively. Wherever it is convenient, we will write 1 for the identity map of any set with itself, and if $\pi$ is a representation of some group $K \ni k$ we will write $\pi_{k}$ for $\pi(k)$ if it abbreviates the notation. We will often use the above filtration where $k$ is the Lie algebra $\mathfrak{h}$ of the group $H$ fixed throughout the paper, and so we will write $\mathfrak{U}_{r}$ for $\mathfrak{U}_{r}(\mathfrak{h})$. We will need to choose a subspace $\mathfrak{r}$ complementary to the subalgebra $\mathfrak{s}$ of $\mathfrak{h}$, and we write $R_{1}$ for the subspace $\mathbb{C} 1 \oplus \mathfrak{r}$ of $\mathfrak{U}_{1}$, where 1 is the identity in $\mathfrak{U}_{0}$.

Definition. Let $H$ be a real Lie group, and let $S$ be a Lie subgroup. A representation of the infinitesimal neighborhood of $S$ in $H$, or more briefly a representation of $S^{\infty}$, is a complex finite dimensional vector space $W_{0}$, a representation $\sigma$ of $S$ on $W_{0}$, an $S$-invariant subspace $W_{1}$ of $W_{0}$, and for each $X \in \mathfrak{h}$ a linear map $\sigma(X): W_{1} \rightarrow W_{0}$ such that:

1. Let $d \sigma: \mathfrak{s} \rightarrow \operatorname{End}\left(W_{0}\right)$ be the differential of $\left.\sigma\right|_{S}$. Then for all $X \in \mathfrak{s}$, $\left.d \sigma(X)\right|_{W_{1}}=\sigma(X)$.

2. For all $s \in S$ and $X \in \mathfrak{h}, \sigma(s) \circ \sigma(X) \circ \sigma\left(s^{-1}\right)=\sigma\left(\operatorname{Ad}_{s} X\right)$ on $W_{1}$.

3. For all $X, Y \in \mathfrak{h},[\sigma(X), \sigma(Y)]=\sigma([X, Y])$ wherever the left hand side is defined, i.e., on the intersection of the inverse images of $W_{1}$ under the maps $\sigma(X)$ and $\sigma(Y)$.

We will often refer to such representations simply as $\sigma, W$, where it is understood that $W$ denotes the flag $W_{0} \supset W_{1}$ and $\sigma$ denotes the actions of both $S$ and $\mathfrak{h}$. The representations of $S^{\infty}$ form a category: a morphism $T$ from an object $\sigma, W$ to an object $\sigma^{\prime}, W^{\prime}$ is a linear map $T: W_{0} \rightarrow W_{0}^{\prime}$ such that $T\left(W_{1}\right) \subset W_{1}^{\prime}$ and $T$ intertwines both the $S$ and $\mathfrak{h}$ actions. There is an obvious notion of subobjects: if $\sigma, W$ is as above and $V=\left\{V_{0} \supset V_{1}\right\}$ is a subflag of $W_{0} \supset W_{1}$, such that $V_{k}$ is an $S$-invariant subspace of $W_{k}$ for $k=0,1$ and $\sigma(X)\left(V_{1}\right) \subset V_{0}$ for all $X \in \mathfrak{h}$, then $V$ is a subobject of $\sigma, W$.

There is also a notion of quotient objects, but only certain subobjects are permitted as factors. Specifically, if $\sigma, W$ and $V$ are as above and $V_{1}=V_{0} \cap$ $W_{1}$, then we define the quotient representation $\bar{\sigma}, W / V$ of $S^{\infty}$ by $(W / V)_{k}=$ $W_{k} / V_{k}$ for $k=0,1$ and $\bar{\sigma}$ the obvious quotient action. The property $V_{1}=$ $V_{0} \cap W_{1}$ is necessary and sufficient to insure that $(W / V)_{1}$ is canonically a subspace of $(W / V)_{0}$, and subrepresentations having it will be said to have the "quotient property." As usual, by a subquotient of a representation $\sigma$ 
of $S^{\infty}$ is meant a quotient of a subrepresentation of $\sigma$, and we have that a subquotient of a subquotient is another subquotient.

If $\pi$ is a representation of $H$ on a space $V$, it restricts to a representation $\left.\pi\right|_{S^{\infty}}$ of $S^{\infty}$ on the flag $V_{0}=V_{1}=V$, and if $\sigma$ is a representation of $S^{\infty}$ on a flag $W_{0} \supset W_{1}$, we define its restriction to $S$ to be the representation $\left.\sigma\right|_{S}$ on $W_{0}$.

Theorem 2.1. Suppose that $H$ is a real algebraic matrix group and $S$ is an algebraic subgroup of $H$, and let $\sigma$ be a representation of $S^{\infty}$ on a flag $W$ whose restriction to $S$ is rational. Then $\sigma$ is isomorphic to a subquotient of the restriction of a finite dimensional rational representation of $H$ to $S^{\infty}$.

Proof. The proof will be in three steps, and contains several lemmas. The idea is to copy the proof of the standard fact that any rational representation $\pi$ of $S$ on a space $V$ is a subquotient of the restriction of a finite dimensional representation of $H$, which goes as follows. Let us write $\mathcal{O} K$ for the complexvalued regular functions on any real algebraic matrix group $K$, and define a map $F: V \rightarrow \mathcal{O} S \otimes V$ by $F(v)(s)=\pi_{s} v$. Then $F$ is an injection and intertwines $\pi$ with $\rho \otimes \mathbf{1}$, where $\rho$ is the right regular representation. Since $\mathcal{O} S$ is a quotient of $\mathcal{O H}, \pi$ is a subquotient of the restriction to $S$ of the representation $\rho \otimes \mathbf{1}$ of $H$ on $\mathcal{O} H \otimes V$. Since $\mathcal{O} H$ has a finite dimensional $H$-invariant filtration, the fact is proven. We remark that nowhere below do we need the third axiom in the definition of representations of $S^{\infty}$.

Step 1. Given a representation $\pi$ of $S$ on a space $V$, write $V \otimes_{\mathfrak{s}} \mathfrak{U}_{n}$ for the quotient $V \otimes_{\mathfrak{U}(\mathfrak{s})} \mathfrak{U}_{n}$ of $V \otimes \mathfrak{U}_{n}$ by $\operatorname{Span}_{\mathbb{C}}\left\{\pi_{X} v \otimes Z+v \otimes X Z: v \in V, X \in \mathfrak{s}, Z \in\right.$ $\left.\mathfrak{U}_{n-1}\right\}$. The representation $\sigma \otimes \operatorname{Ad}$ of $S$ factors through to a representation $\sigma \otimes_{\mathfrak{s}}$ Ad of $S$ on $V \otimes_{\mathfrak{s}} \mathfrak{U}_{n}$, which we extend to a representation $\pi^{(n)}$ of $S^{\infty}$ on the flag $V \otimes_{\mathfrak{s}} \mathfrak{U}_{n} \supset V \otimes_{\mathfrak{s}} \mathfrak{U}_{n-1}$ by defining $\left.\pi^{(n)}\right|_{\mathfrak{h}}$ to be $\mathbf{1} \otimes \rho$, where $\rho$ is the right regular representation of $\mathfrak{h}$ on $\mathfrak{U}(\mathfrak{h})$. In other words, if $v \otimes Z \in V \otimes_{\mathfrak{s}} \mathfrak{U}_{n-1}$ and $X \in \mathfrak{h}$, then $\pi_{X}^{(n)}(v \otimes Z)=-v \otimes Z X$. One checks that $\pi^{(n)}$ is indeed a representation of $S^{\infty}$.

Lemma 2.2. Given any representation $\sigma$ of $S^{\infty}$ on a flag $W$, let $\pi$ be the representation $\left.\sigma\right|_{S}$ on $W_{0}$. Then $\sigma$ is isomorphic to a subquotient of the representation $\pi^{(1)}$ of $S^{\infty}$ on $W_{0} \otimes_{\mathfrak{s}} \mathfrak{U}_{1} \supset W_{0} \otimes_{\mathfrak{s}} \mathfrak{U}_{0}$.

Proof. Identify $W_{0} \otimes_{\mathfrak{s}} \mathfrak{U}_{0}$ with $W_{0}$, and define a subspace $K=\operatorname{Span}_{\mathbb{C}}\left\{\sigma_{X} w+\right.$ $\left.w \otimes X: w \in W_{1}, X \in \mathfrak{h}\right\}$ of $W_{0} \otimes_{\mathfrak{s}} \mathfrak{U}_{1}$. Using the fact that $\sigma$ is a representation of $S^{\infty}$ we find that $K$ is $S$-invariant, and so $K \supset 0$ is an $S^{\infty}$-subrepresentation of $\pi^{(1)}$. Now it is standard that the quotient map $W_{0} \otimes \mathfrak{U}_{1} \rightarrow W_{0} \otimes_{\mathfrak{s}} \mathfrak{U}_{1}$ restricts to a linear isomorphism from $W_{0} \otimes R_{1}$ to $W_{0} \otimes_{\mathfrak{s}} \mathfrak{U}_{1}$, where $R_{1}=\mathfrak{U}_{0} \oplus \mathfrak{r}$ was defined at the beginning of this section. 
It follows that the map $W_{1} \otimes \mathfrak{r} \rightarrow K$ defined by $w \otimes Y \mapsto \sigma_{Y} w+w \otimes Y$ is a linear isomorphism, and that $K \cap W_{0}=0$. Therefore $K \supset 0$ has the quotient property, and furthermore, the linear map from $W_{0}$ to $W_{0} \otimes_{\mathfrak{s}} \mathfrak{U}_{1} / K$ defined by inclusion from $W_{0}$ to $W_{0} \otimes_{\mathfrak{s}} \mathfrak{U}_{1}$ followed by projection is injective. It is easy to prove that it is an $S^{\infty}$-intertwining map, which proves the lemma.

Step 2. In light of Lemma 2.2, Theorem 2.1 will be proven if we prove it for the special case that $\sigma$ is the representation $\pi^{(1)}$ of $S^{\infty}$ on $V \otimes_{\mathfrak{s}} \mathfrak{U}_{1} \supset V$, for some rational representation $\pi$ of $S$ on $V$. In order to adapt to this setting the proof that rational representations of $S$ are subquotients of restrictions of representations of $H$, it is helpful to consider duals of representations of $S^{\infty}$, despite the fact that they are not themselves representations of $S^{\infty}$.

If $\sigma$ is a representation of $S^{\infty}$ on $W_{0} \supset W_{1}$, we define its dual $\sigma^{*}$ to be the following collection of objects: The spaces $W_{0}^{*}$ and $W_{1}^{*}$, the canonical projection $p_{W}: W_{0}^{*} \rightarrow W_{1}^{*}$, the representations of $S$ on $W_{0}^{*}$ and $W_{1}^{*}$ dual to $\left.\sigma\right|_{S}$ (both denoted by $\sigma^{*}$ ), and finally the maps $\sigma_{Y}^{*}=-\sigma_{Y}^{T}: W_{0}^{*} \rightarrow W_{1}^{*}$ for all $Y \in \mathfrak{h}$, where $T$ denotes the transpose. Note that $p_{W}$ is an $S$-map, and axioms 1 and 2 in the definition of representations of $S^{\infty}$ translate to $p_{W} \circ d \sigma_{X}^{*}=\sigma_{X}^{*}$ for $X \in \mathfrak{s}$, and $\sigma_{s}^{*} \circ \sigma_{Y}^{*} \circ \sigma_{s^{-1}}^{*}=\sigma^{*}\left(\operatorname{Ad}_{s} Y\right)$ for $s \in S$ and $Y \in \mathfrak{h}$. We shall refer to $\sigma^{*}$ as acting in $p_{W}: W_{0}^{*} \rightarrow W_{1}^{*}$. The following lemma says that if a map intertwines the duals of two representations of $S^{\infty}$, then its transpose intertwines the representations themselves; we omit the proof.

Lemma 2.3. Let $(\mu, M)$ and $(\nu, N)$ be representations of $S^{\infty}$, and for $i=$ 0,1 suppose that $T_{i}: N_{i}^{*} \rightarrow M_{i}^{*}$ are maps such that $p_{M} \circ T_{0}=T_{1} \circ p_{N}$, both $T_{i}$ intertwine the representations $\nu^{*}$ and $\mu^{*}$ of $S$, and $T_{1} \circ \nu_{Y}^{*}=\mu_{Y}^{*} \circ T_{0}$ : $N_{0}^{*} \rightarrow M_{1}^{*}$ for all $Y \in \mathfrak{h}$. Then the map $T^{*}=T_{0}^{T}: M_{0} \rightarrow N_{0}$ intertwines the representations $\mu$ and $\nu$ of $S^{\infty}$, and if $T_{0}$ is injective or surjective then $T^{*}$ is surjective or injective, respectively.

For any representation $\beta$ of $S$ on a space $B$, let us define a space

$$
\mathcal{H}_{B}=\operatorname{Hom}_{\mathfrak{s}}\left(\mathfrak{U}_{1}, B\right)=\left\{F: \mathfrak{U}_{1} \rightarrow B: F(X)=\beta_{X} F(1) \forall X \in \mathfrak{s}\right\} .
$$

This is the space of maps intertwining the left action $\lambda_{X}: \mathfrak{U}_{0} \rightarrow \mathfrak{U}_{1}$ with the action of $\beta_{X}$ on $B$ for all $X \in \mathfrak{s}$. The reader is warned that since $\mathfrak{U}_{1}$ is not closed under $\lambda$, this is not the standard use of the notation $\mathrm{Hom}_{\mathfrak{s}}$. In fact, the restriction map from $\mathcal{H}_{B}$ to $\operatorname{Hom}_{\mathbb{C}}\left(R_{1}, B\right)$ is a linear isomorphism. 
For any $Z \in \mathfrak{U}_{1}$, let $e_{Z}: \mathcal{H}_{B} \rightarrow B$ be the evaluation map $F \mapsto F(Z)$. Note that $\mathcal{H}_{V^{*}}$ is dual to $V \otimes_{\mathfrak{s}} \mathfrak{U}_{1}$ by the pairing $\langle F, v \otimes Z\rangle=\langle F(Z), v\rangle$, and the representation $\operatorname{Hom}\left(\mathrm{Ad}, \pi^{*}\right)$ of $S$ on $\mathcal{H}_{V^{*}}$ is dual to $\pi \otimes_{\mathfrak{s}} \mathrm{Ad}$. It is an exercise to check that the dual $\pi^{(1) *}$ of the representation $\pi^{(1)}$ of $S^{\infty}$ acts in $e_{1}: \mathcal{H}_{V^{*}} \rightarrow V^{*}$, with $S$-actions $\operatorname{Hom}\left(\mathrm{Ad}, \pi^{*}\right)$ and $\pi^{*}$, and $\mathfrak{h}$-action $\pi_{Y}^{(1) *}=e_{Y}: \mathcal{H}_{V^{*}} \rightarrow V^{*}$.

Now let $\mathcal{O}_{1} H \subset \mathcal{O}_{2} H \subset \cdots \mathcal{O} H$ be any finite dimensional filtration on the regular functions $\mathcal{O} H$ that is invariant under the right regular representation $\rho$, and let $\left\{\mathcal{O}_{k} S\right\}$ be the $\left.\rho\right|_{S}$-invariant filtration on $\mathcal{O} S$ given by restricting it. Let us write simply $\mathcal{H}$ for the space $\mathcal{H}_{\mathcal{O}}$, defined using the representation $\rho$ of $S$ on $\mathcal{O} S$. Then the spaces $\mathcal{H}_{k}=\mathcal{H}_{\mathcal{O}_{k} S}$ give a finite dimensional $S$ invariant filtration of $\mathcal{H}$.

Let $\mathcal{O}_{k}^{*} S$ be the vector space dual to $\mathcal{O}_{k} S$, and let $\rho_{k}^{*}$ be the dual of the right regular representation on it. Then the representation $\rho_{k}^{*(1)}$ of $S^{\infty}$ acts in $\mathcal{O}_{k}^{*} S \otimes_{\mathfrak{s}} \mathfrak{U}_{1} \supset \mathcal{O}_{k}^{*} S$, and we will write $\mu_{k}$ for its dual. We have seen that $\mu_{k}$ acts in $e_{1}: \mathcal{H}_{k} \rightarrow \mathcal{O}_{k} S$, with $\left.\mu_{k}\right|_{S}=\operatorname{Hom}(\operatorname{Ad}, \rho)$ and $\mu_{k}(Y)=e_{Y}$ for $Y \in \mathfrak{h}$.

Lemma 2.4. Let $\pi$ be a rational representation of $S$ on a space $V$. Then for $k$ large enough, the representation $\pi^{(1)}$ of $S^{\infty}$ is isomorphic to a quotient of the representation $\rho_{k}^{*(1)} \otimes 1$ of $S^{\infty}$ on $\left(\mathcal{O}_{k}^{*} S \otimes_{\mathfrak{s}} \mathfrak{U}_{1}\right) \otimes V \supset \mathcal{O}_{k}^{*} S \otimes V$, whose action on the factor $V$ is trivial.

Proof. Define maps $T_{0}: \mathcal{H}_{V^{*}} \rightarrow \mathcal{H} \otimes V^{*}$ and $T_{1}: V^{*} \rightarrow \mathcal{O} S \otimes V^{*}$ as follows. For $F \in \mathcal{H}_{V^{*}}, T_{0} F: \mathfrak{U}_{1} \rightarrow \mathcal{O} S \otimes V^{*}$ has for its value at $Z$ the $V^{*}$-valued rational function on $S$ defined by $T_{0} F(Z)(s)=\pi_{s}^{*} F(Z)$, and for $\omega \in V^{*}$, $T_{1} \omega$ is the $V^{*}$-valued rational function $s \mapsto \pi_{s}^{*} \omega$ on $S$.

Since $\pi$ is rational, $T_{0} F(Z)$ and $T_{1} \omega$ are indeed rational functions, and both $T_{0}$ and $T_{1}$ are clearly injective. Since $\mathcal{H}_{V^{*}}$ is finite dimensional, the image of $T_{0}$ lies inside $\mathcal{H}_{k} \otimes V^{*}$ if we take $k$ large enough. Then checking the definitions shows that the $T_{i}$ satisfy the hypotheses of Lemma 2.3 with respect to $\pi^{(1) *}$ and $\mu_{k} \otimes \mathbf{1}$, and so $T^{*}=T_{0}^{T}:\left(\mathcal{O}_{k}^{*} S \otimes_{\mathfrak{s}} \mathfrak{U}_{1}\right) \otimes V \rightarrow V \otimes_{\mathfrak{s}} \mathfrak{U}_{1}$ is a surjective $S^{\infty}$-map. As was proven in Lemma 1.1 of [5], this means that $\operatorname{kernel}\left(T^{*}\right)$ is an $S^{\infty}$-subrepresentation of $\rho_{k}^{*(1)} \otimes \mathbf{1}$ with the quotient property, and $\pi^{(1)}$ is isomorphic to the resulting quotient.

Step 3. In light of Lemmas 2.2 and 2.4, the next two lemmas complete the proof of Theorem 2.1.

Lemma 2.5. For any $k>0$, there exists some $j>0$ such that the representation $\rho_{k}^{*(1)}$ of $S^{\infty}$ on $\mathcal{O}_{k}^{*} S \otimes_{\mathfrak{s}} \mathfrak{U}_{1} \supset \mathcal{O}_{k}^{*} S$ is isomorphic to a subrepresentation of the restriction to $S^{\infty}$ of the dual $\rho_{j}^{*}, \mathcal{O}_{j}^{*} H$ of the right regular representation $\rho$ of $H$ on $\mathcal{O}_{j} H$. 
Proof. Let $r: \mathcal{O} H \rightarrow \mathcal{O} S$ be the restriction map, and define a map $\alpha$ : $\mathcal{O} H \rightarrow \mathcal{H}$ by $\alpha f(Z)=r \circ \rho_{Z}(f)$ for all $Z \in \mathfrak{U}_{1}$. We will see in Lemma 2.6 that $\alpha$ is surjective, and so $\mathcal{H}_{k} \subset \alpha\left(\mathcal{O}_{j} H\right)$ for some $j$. On the other hand, $\alpha\left(\mathcal{O}_{j} H\right) \subset \mathcal{H}_{j}$. Now the restriction $\left.\rho_{j}^{*}\right|_{S^{\infty}}$ of the representation $\rho_{j}^{*}$ of $H$ on $\mathcal{O}_{j}^{*} H$ acts in the trivial flag $\mathcal{O}_{j}^{*} H \supset \mathcal{O}_{j}^{*} H$, and we will write $\nu_{j}$ for its dual, which acts in $\mathbf{1}: \mathcal{O}_{j} H \rightarrow \mathcal{O}_{j} H$. Recall that we are writing $\mu_{j}$ for the dual of $\rho_{j}^{*(1)}$, and that $\mu_{j}$ acts in $e_{1}: \mathcal{H}_{j} \rightarrow \mathcal{O}_{j} S$.

Define maps $T_{0}: \mathcal{O}_{j} H \rightarrow \mathcal{H}_{j}$ and $T_{1}: \mathcal{O}_{j} H \rightarrow \mathcal{O}_{j} S$ by $T_{0}=\alpha$ and $T_{1}=r$. One checks that these maps satisfy the hypotheses of Lemma 2.3 with respect to $\nu_{j}$ and $\mu_{j}$, and so $T^{*}=T_{0}^{T}: \mathcal{O}_{j}^{*} S \otimes_{\mathfrak{s}} \mathfrak{U}_{1} \rightarrow \mathcal{O}_{j}^{*} H$ intertwines $\rho_{j}^{*(1)}$ and $\left.\rho_{j}^{*}\right|_{S^{\infty}}$. Since the image of $T_{0}$ contains $\mathcal{H}_{k}$, the restriction of $T^{*}$ to $\mathcal{O}_{k}^{*} \otimes_{\mathfrak{s}} \mathfrak{U}_{1}$ is injective, which completes the proof.

For the last lemma, recall that $S$ is an algebraic subgroup of the real algebraic matrix group $H$, and that we are writing $\mathcal{O} K$ for the complexvalued regular functions on any real algebraic matrix group $K$. Let $K_{c}$ be the complex algebraic group whose real points make up $K$; then the algebra of regular functions $\mathcal{O} K_{c}$ on $K_{c}$ is equal to $\mathcal{O} K$. Define $\mathcal{H}$ and $\alpha: \mathcal{O} H_{c} \rightarrow \mathcal{H}$ as above.

Lemma 2.6. The map $\alpha$ is surjective.

Proof. Let $e_{Z}: \mathcal{H} \rightarrow \mathcal{O} S_{c}$ be evaluation at $Z \in \mathfrak{U}_{1}$, and note that $e_{1} \circ \alpha=r$ is surjective. Hence it is enough to prove that $\operatorname{kernel}\left(e_{1}\right) \subset \operatorname{image}(\alpha)$. Let $\mathcal{I} \subset \mathcal{O} H_{c}$ be the ideal vanishing on $S_{c}$, and let $\mathcal{J}=\operatorname{kernel}\left(e_{1}\right)$. If $F \in \mathcal{J}$, $F(X)=\rho_{X} F(1)=0$ for all $X \in \mathfrak{s}$, from which it follows that $\mathcal{J}$ is isomorphic to $\operatorname{Hom}_{\mathbb{C}}\left(\mathfrak{h} / \mathfrak{s}, \mathcal{O} S_{c}\right)=(\mathfrak{h} / \mathfrak{s})^{*} \otimes \mathcal{O} S_{c}$, the global sections of the conormal bundle of $S_{c}$ in $H_{c}$. Now note that $\alpha\left(\mathcal{I}^{2}\right)=0$ and $\alpha(\mathcal{I}) \subset \mathcal{J}$, so $\alpha$ factors through to $\bar{\alpha}: \mathcal{I} / \mathcal{I}^{2} \rightarrow(\mathfrak{h} / \mathfrak{s})^{*} \otimes \mathcal{O} S_{c}$. But this is just the first map in the conormal exact sequence, which is well-known to be an isomorphism of sheaves when $S_{c}$ is connected. Here it is an isomorphism of global sections if $S_{c}$ is connected, because $S_{c}$ is a closed subvariety of the affine variety $H_{c}$. An easy argument shows that it is still surjective if $S_{c}$ is not connected.

\section{References}

[1] G. Cassinelli, G. Olivieri, P. Truini and V.S. Varadarajan, On some nonunitary representations of the Poincaré group and their use for the construction of free quantum fields, J. Math. Phys., 30 (1989), 2692-2707.

[2] G. Casinelli, P. Truini and V.S. Varadarajan, Hilbert space representations of the Poincaré group for the Landau gauge, J. Math. Phys., 32 (1991), 1076-1090. 
[3] C. Conley, Representations of finite length of semidirect product Lie groups, J. Funct. Anal., 114 (1993), 421-457.

[4] Little group method for smooth representations of finite length, Duke Math. J., 79(3) (1995), 619-666.

[5] Geometric realizations of representations of finite length, Reviews Math. Phys., 9(7) (1997), 821-851.

[6] A. Guichardet, Extensions des représentations induites des produits semidirects, J. für Reine Angew. Math., 310 (1979), 7-32.

[7] Représentations de longeur finie des groupes de Lie inhomogènes, Astérisque, 124-125 (1985), 212-252.

[8] G. Rideau, Noncompletely reducible representations of the Poincaré group associated with the Lorentz gauge, J. Math. Phys., 19 (1978), 1627-1634.

Received October 10, 1996.

University of North Texas

DENTON, TX 76203-5116

E-mail address: conley@unt.edu 